\title{
SCIENCE, TÉLÉVISION ET RATIONALITÉ
}

Igor Babou (École Normale supérieure Lettres et Sciences Humaines)

La télévision participe à un processus de circulation sociale des connaissances scientifiques dont les enjeux sont souvent appréhendés en termes quantitatifs : il suffirait que la télévision diffuse «plus de science » pour permettre au public de mieux comprendre le discours des chercheurs. Cet article va montrer que pour comprendre ce processus et ses enjeux, il importe de complexifier l'étude de la médiatisation des sciences en décrivant le «langage » télévisuel dans lequel cette vulgarisation s'incarne, et les cadres interprétatifs qui permettent d'appréhender la science à travers un imaginaire et des valeurs. Au-delà de la représentation et de la mise en circulation de connaissances, la vulgarisation témoigne en effet des enjeux sociaux de la rationalité scientifique.

L'étude des métaphores constitue un analyseur de choix pour repérer des représentations et des enjeux dans les discours sociaux. C'est par cette méthode que l'on étudiera les relations entre les formes du discours télévisuel de vulgarisation et les représentations sociales de la science qui s'y articulent.

\section{Science, vulgarisation et métaphores}

En dehors des dictionnaires, s'il y a un type de discours réputé réfractaire à toute métaphorisation, c'est bien le discours scientifique : en principe, tous ses énoncés doivent être précisément interdéfinis, sans la moindre ambiguïté. Ce n'est pourtant pas toujours le cas : de la théorie du « chaos » à la représentation planétaire de l'atome, en passant par divers procédés d'analogie, l'histoire des terminologies et des concepts scientifiques est riche de métaphores ${ }^{1}$. Jacobi évoque ainsi le cas de la géophysique dont un dictionnaire spécialisé classe des métaphores parmi d'autres termes scientifiques ${ }^{2}$. Les sciences sociales sont elles aussi à l'origine de nombreuses analogies : l' « acteur social » joue son « rôle », ses énoncés sont des « actes » de langage, la communication est tantôt « orchestrale », tantôt « télégraphique », les médias sont crédités d'un «pouvoir » quand ils n'ont pas un « impact», des « contrats » de lecture ou des «promesses » relient leurs discours à leurs destinataires ${ }^{3}$, etc. Des sciences de la nature aux sciences sociales, il convient alors de se demander si la recherche mobilise des systèmes conceptuels, des méthodes et des données d'observation derrière ces métaphores. La métaphore devient ainsi autre chose qu'un simple beseturantudusdiskourdgarisation critiquent souvent les métaphores médiatiques sur la base d'une représentation idéalisée du discours scientifique, celui-ci devant être exempt d'analogie ou de métaphore. La métaphorisation des concepts scientifiques, leur ontologisation est décriée par Roqueplo ${ }^{4}$ ou Allemand ${ }^{5}$ comme caractéristique des pratiques de vulgarisation : elles rabattraient les concepts scientifiques sur le sens commun du public. Jurdant explique que, dans la presse de vulgarisation, « La métaphore est à l'origine de paradigmes particuliers des-

\footnotetext{
' Par exemple, Léonard De Vinci illustrant dans ses Carnets une dissection de tête humaine à l'aide d'une analogie avec la tructure d'un oignon (VINCI, Léonard (de), Carnets - Tome I, Paris, Gallimard, 1942, p. 185). Ce procédé de l'analogie explicative était d'ailleurs fréquent pour lui.

2 JACOBI, Daniel, La communication scientifique, Grenoble, P.U.G, 1999, p. 85-86.

${ }^{3}$ La notion de «contrat » de communication est développée par VÉRON, Éliseo, L'analyse du «contrat de lecture »: une nouvelle méthode pour les études de positionnement des supports de presse, in : Les médias - Expériences, recherches actuelles, applications, Paris, IREP, 1985, p. 203-229. Cette notion est fortement critiquée, au motif qu'elle serait métaphorique, par JOST, François, Le genre télévisuel. Du contrat à la promesse, in Degrés $n^{\circ}$ 94, Bruxelles, ASBL Degrés, 1998, p. 1-20.

${ }^{4}$ ROQUEPLO, Philippe, Le partage du savoir, Paris : Seuil, 1974.

${ }^{5}$ ALLEMAND, Étienne, L’information scientifique à la télévision, Paris, Anthropos, 1983.
} 
tinés à rendre possible l'intégration des signifiants scientifiques à l'intérieur de la langue $»^{6}$. Gravitant autour d'un nombre limité d'axes d'opposition, les métaphores du pulsar qu'il relève dans des revues

[...] sont destinées à permettre au lecteur de se faire des idées manipulables en tant qu'idées à l'intérieur d'une logique préexistante. La vulgarisation provoque ainsi une augmentation purement qualitative du capital d'idées disponibles sans en changer l'agencement qualitatif. [...] Il s'agit donc bien d'une application de la formule du savoir ce que l'on savait déjà, dans la mesure où les règles d'utilisation du capital d'idées restent inchangées quel que soit le niveau quantitatif de ce capital. Ce sont ces règles d'utilisation dont la vulgarisation s'oblige à assurer la permanence — celle-ci ayant été mise en question pour un moment, celui de la découverte scientifique — qui constituent l'idéologie à proprement parler ${ }^{7}$.

Lorsqu'elle n'est pas décrite comme une opération idéologique de construction du mythe de la scientificité (paradigme de la trahison), la vulgarisation est vue comme la traduction d'un discours ésotérique en une langue plus accessible : le paradigme du troisième homme constitue ainsi une représentation classique du rôle des journalistes ${ }^{8}$. Parfois engagés dans une logique didactique (les métaphores auraient pour fonction de faciliter la compréhension des concepts en opérant une traduction basée sur le sens commun), les journalistes peuvent aussi revendiquer une fonction poétique en considérant qu'ils ont la charge non d'expliquer les sciences, mais d'élaborer un spectacle, une dramaturgie'.

Idéologique ? Didactique ? Poétique ? Les analyses qui abordent les discours médiatiques à propos de science et leurs métaphores en mobilisant la linguistique ou le fonctionnalisme sociologique (réduire une pratique culturelle à une fonction sociale unique et stable) semblent insuffisantes pour décrire le fonctionnement et les enjeux d'un champ de pratiques aussi hétérogènes et contradictoires. De Moscovici ${ }^{10}$ à Jeanneret ${ }^{11}$ en passant par Fouquier et Véron ${ }^{12}$, Cheveigné ${ }^{13}$ ou Jurdant ${ }^{14}$, nombreux sont les chercheurs qui ont abordé les discours à propos de science de manière plus globale, afin d'en saisir la diversité des formes et des enjeux. Aujourd'hui, il semble acquis que l'on ne peut plus se contenter de réduire cette diversité à des fonctions sociale unitaires et stables (aussi commodes soient-elles pour l'argumentation et le classement de tel ou tel chercheur dans les paradigmes correspondants), mais que la recherche en communication doit en détailler les mécanismes afin d'en faire apparaître la complexité.

\section{La rationalité scientifique}

Au delà de l'information sur les découvertes scientifiques, comment la télévision représente-telle l'idée même de rationalité scientifique ? Ce concept indissociable de celui de raison, a donné lieu à diverses définitions contradictoires dans les écrits épistémologiques. En analysant un corpus de dictionnaires généralistes ou philosophiques, ainsi que des textes de philosophie et d'histoire des sciences ${ }^{15}$, on voit apparaître non pas un dénominateur commun mais un ensemble d'axes d'interrogations et de figures du discours : un processus historique et social de légitimation, de construction ou de critique des critères de scientificité. On con-

\footnotetext{
${ }^{6}$ JURDANT, Baudoin, Vulgarisation scientifique et idéologie, in Communications n 14, Paris, Seuil, 1969, p. 157.

${ }^{7}$ Ibid., p. 158.

${ }^{8}$ MOLES, A. et OULIF, J. M., Le troisième homme, vulgarisation scientifique et radio, in Diogène $n^{\circ}$ 58, 1967, p. 29-40.

${ }^{9}$ TRISTANI-POTTEAUX, Françoise, Les journalistes scientifiques, Paris : Economica, 1997, p. 44-48.

${ }^{10}$ MOSCOVICI, Serge, La psychanalyse, son image et son public, Paris, PUF, 1976.

"JEANNERET, Yves, Écrire la science, Paris, PUF, 1994.

${ }^{12}$ FOUQUIER, Éric et VÉRON, Éliseo, Les spectacles scientifiques télévisés, Paris, La Documentation Française, 1985.

${ }^{13}$ CHEVEIGNÉ, Suzanne (de), L'environnement dans les journaux télévisés, Paris, CNRS Éditions, 2000.

${ }^{14}$ JURDANT, Baudoin, Impostures scientifiques, Paris, La Découverte/Alliage, 1998.

${ }^{15}$ Babou, Igor, Science, télévision et rationalité - Analyse du discours télévisuel à propos du cerveau - Thèse de doctorat en Sciences de la Communication sous la co-direction de Carmen Compte et Suzanne de Cheveigné, Paris, Université Paris 7, 1999.
} 
state que la rationalité scientifique est généralement considérée comme une procédure (méthode, moyens) mise en œuvre sur le « réel » par un sujet pensant à partir d'axiomes logiques et visant une fin (la vérité, la connaissance) attestée par un public (l'intersubjectivité permettant une construction collective des faits). Cette définition sommaire, forcément schématisée, constitue le noyau conceptuel des définitions de la rationalité scientifique.

Ce concept mobilise ensuite des représentations associées repérables dans les textes qui ont contribué à le définir. Des attributs viennent en effet se greffer sur le noyau conceptuel de la rationalité et se constituent en système en fonction duquel les auteurs adoptent des positions différentes. Il s'agit d'un ensemble d'axiologies, c'est-à-dire, selon Greimas ${ }^{16}$, d'axes d'opposition sur lesquels s'organisent des valeurs. D'après cet auteur ${ }^{17}$, on peut définir l'idéologie comme l'organisation récurrente au sein d'un discours de valeurs (positives ou négatives) associées aux «pôles » de ces axes d'opposition. Lors de l'analyse du corpus des dictionnaires et des textes d'histoire et philosophie des sciences, on a pu repérer les six axiologies suivantes :

Libre arbitre - domination

Homme - animal

Esprit - corps

Raison - opinion

Raison - croyance

Réductionnisme - holisme

Ces axes d'opposition structurent fortement le discours sur la rationalité et constituent des lieux communs du discours sur la rationalité scientifique et la raison du sujet pensant. Autour de ces oppositions s'organisent des valeurs (morales, politiques, philosophiques ou religieuses) mobilisées dans le champ des débats sur la science.

\section{Un corpus d'émissions sur le cerveau}

L'hypothèse présentée ici consiste à dire que si les réflexions sur la raison et la rationalité ont occupé l'histoire des idées durant plusieurs siècles, on doit en trouver des traces dans le discours télévisuel à propos de science : ces traces témoigneraient d'un ensemble de représentations sociales inscrites dans des schémas de pensée collectifs, des manières d'agir et des discours partagés. Diffusées largement par la scolarisation, les représentations de la rationalité ne restent en effet pas cloisonnées dans les traités d'épistémologie. L'ampleur de l'affaire Sokal est là pour rappeler que la question de la scientificité est un enjeu majeur du débat public contemporain ${ }^{18}$.

Pour se donner les moyens de vérifier comment la rationalité est représentée par la télévision, on a constitué un corpus d'émissions. Ce corpus a été recueilli à l'Inathèque de France qui gère le dépôt légal des chaînes et dispose d'une base de données permettant d'accéder aux émissions diffusées par les chaînes hertziennes. La thématique de la rationalité n'ayant pas été traitée de manière explicite par la télévision, on a travaillé autour de la thématique du cerveau pour favoriser l'émergence de représentations implicites. On a sélectionné 56 émissions diffusées entre 1975 et 1994, soit près de vingt années d'émissions sur le cerveau. Le corpus comprend trois tranches $(1975$ à 1982, 1987, 1994) et se compose de 26 « sujets » du journal télévisé, de 17 documentaires et de 13 magazines. Toutes ces émissions ont été visionnées, leurs images imprimées et leurs textes et commentaires retranscrits. C'est à l'aide de ce corpus qu'a été menée l'analyse des axiologies, ces jeux d'opposition binaires qui structurent le discours sur la rationalité, et dont une partie va être présentée maintenant.

\footnotetext{
${ }^{16}$ Greimas, A. J. et Courtès, J., Sémiotique — Dictionnaire raisonné de la théorie du langage, Paris, Hachette, 1993, p. 25.

${ }^{17}$ Ibid., p. 179.

${ }^{18}$ JEANNERET, Yves, L'affaire Sokal ou la querelle des impostures, Paris, PUF, 1998 ; JURDANT, Baudoin, Impostures scientifiques, Paris, La Découverte/Alliage, 1998.
} 


\section{Libre arbitre et domination}

De Descartes aux Lumières et jusqu'au XIX ${ }^{\mathrm{e}}$ siècle, la rationalité est considérée comme un facteur d'émancipation et de liberté qui prend la forme d'une quête de la vérité scientifique. Mais à partir du $\mathrm{XX}^{\mathrm{e}}$ siècle, la rationalité commence à être pensée bien différemment. Pour Weber $^{19}$, la rationalité instrumentale témoigne d'un élargissement du champ de l'activité rationnelle qui dépasse l'activité scientifique pour s'appliquer au champ social et politique. Pour Russell, «Ce que nous appelons la Raison a un sens parfaitement clair et précis. Cela signifie le choix des moyens adéquats à une fin que l'on désire atteindre. Cela n'a absolument rien à voir avec le choix des fins $»^{20}$. Pour Simon également, « La raison est pleinement instrumentale. Elle est incapable de nous dire où nous devons aller; le mieux qu'elle puisse faire, c'est de nous dire comment y aller $»^{21}$. Ces définitions insistent sur l'aspect procédural de la rationalité et en évacuent toute préoccupation éthique. Le concept de rationalité instrumentale peut ainsi se dégager de celui de rationalité scientifique et du contexte qui l'a vu naître (la quête de la vérité comme fin) pour aborder n'importe quel domaine de la vie sociale (l'économique et le politique en particulier). Si un auteur comme Russell conserve la nécessité de l'application d'une volonté du sujet, pour Simon la rationalité semble comme dictée de l'extérieur au sujet, un peu comme si la structure en place ne pouvait faire autre chose qu'orienter les acteurs. On est donc à l'opposé de la notion cartésienne de libre arbitre. Ainsi, une partie du champ épistémologique de la rationalité se développe autour de l'axe « libre arbitre - domination ». Une position sur cet axe caractérise, pour celui qui la prend, le mode d'implication du concept dans l'action individuelle ou sociale.

Le discours télévisuel à propos du cerveau représente-t-il la science comme une entreprise hégémonique menaçant le libre arbitre des individus ou le fonctionnement social ? Ou, au contraire, la représente-t-il comme une garantie de liberté ? On repère ces oppositions dans diverses émissions qui se répartissent sur les vingt années du corpus ${ }^{22}$. Voilà par exemple comment un informaticien spécialiste de la miniaturisation considère les effets de l'avancée technologique en 1987 :

François Mizzi : «[...] tous ces appareils vont simplifier notre vie et nous donner plus de liberté en élaguant une partie de nos tâches intellectuelles répétitives. Que va-t-on faire de cette liberté ? Certains vont bien en

vivre, mais d'autres vont continuer à développer la puissance de ces appareils et cela finit par être dangereux »

La science est dépeinte, assez classiquement, comme une boîte de Pandore d'où peuvent sortir aussi bien la liberté que la domination.

Voici ensuite comment Monique Le Poncin, une neuropsychologue, répond à de jeunes élèves dans une émission éducative diffusée en 1987 :

Élève [par téléphone] : «Bonjour madame. On voudrait savoir aussi... Des connaissances trop importantes sur le cerveau ne pourraient-elles pas nuire à l'homme ? »

\footnotetext{
${ }^{19}$ WEBER, Max, Essais sur la théorie de la science, Paris, Plon, 1968.

${ }^{20}$ Cité par DUPUY, Jean-Pierre, LIVET, Pierre \& Al, Les limites de la rationalité — Tome I : Rationalité, éthique et cognition - Colloque de Cérisy, Paris, La Découverte, 1997, p. 14.

${ }^{21}$ Ibid., p. 14.

${ }^{22}$ Les scientifiques répondent : qu'est-ce qu'un comportement? (documentaire diffusé le 23.07.75 à 22h50 sur TF1); Le propre de l'homme : le cerveau (documentaire de la collection « Histoire de la vie » diffusé le 18.11.1982 à 22h50 sur TF1. Ce documentaire a été ensuite rediffusé trente fois sur TF1 entre 1988 et 1994); L'homme électronique (magazine de la collection « Dimension 3 » diffusé le 06.04.1987 à 22h36 sur FR3); Temps X : Professeur Delgado (diffusé le 02.05.1987 à 16h41 sur TF1); Un univers, l'homme (Collection « Génération 3 », séquence « Matière grise », diffusé le 18.01.94 à 9h53 sur France 3); Nimbus : la mémoire (diffusé le 21.10.94 à $23 \mathrm{~h} 25$ sur France 3) ; Nimbus : Jean-Didier Vincent (diffusé le 18.11.94 à 23h25 sur France 3)
} 
Monique Le Poncin : «Oui. Justement, mais c'est le problème de toute la science, qu'elle soit cérébrale ou qu'elle soit autre. Comme je vous le disais tout à l'heure, entre zéro et trois ans on peut faire des génies. Ca serait catastrophique... [...] On pourrait tout à fait conditionner par les techniques de gestion mentale tel individu, de telle façon à lui faire faire telle ou telle chose. Donc, c'est sûr que c'est très dangereux et que la progression dans la connaissance du cerveau peut amener à des manipulations cérébrales, et il faut que les gens qui s'occupent de ce genre de techniques soient très éthiques »

On trouve le même type d'évocation du problème de la manipulation mentale dans

« Nimbus : la mémoire », qui expose le cas d'une jeune américaine (Holly Ramona) à qui des psychopédagogues auraient «implanté » de faux souvenirs d'inceste. Mais les journalistes qui évoquent ce cas le font dans des termes bien moins catastrophistes que les scientifiques interrogés en 1987. En effet, si l'affaire Ramona est présentée comme scandaleuse, on n'observe aucune mise en accusation de la science. C'est même presque le contraire qui se passe, puisque le cas de Ramona ayant été présenté en introduction du reportage, la question est ainsi posée : « Mais comment de telles manipulations de mémoire sont-elles possibles ?». Et dans ce « comment » ne rentre aucune interrogation éthique, mais plutôt une demande d'explication sur les mécanismes de la mémoire, le cas de Ramona permettant alors aux journalistes d'interroger divers experts de la mémoire : hypnothérapeutes, psychologues ou sociologues. Ce traitement particulièrement modéré, en 1994, d'une thématique présentée de manière beaucoup plus critique en 1987 pourrait indiquer une corrélation entre modalités de légitimation de la science à la télévision et représentation de la rationalitée ${ }^{2}$. Par l'examen de la prochaine occurrence, on va confirmer que l'axiologie « libre arbitre - domination » est associée, en 1994, à un regain d'optimisme.

Chercheur : «On ne dispose pas, et on ne disposera jamais de moyens de prédire à un instant donné, compte tenu de la diversité des interactions qui ont trait à la nature changeante de l'environnement, on ne pourra pas prédire le comportement de tel ou tel individu dans telle ou telle situation. C'est... c'est... heu... le petit reste de liberté individuelle qui peut rester à chacun d'entre nous »

Commentaire off : «Et pour explorer cette part de liberté dont le fonctionnement échappe encore aux sciences exactes, certains chercheurs proposent une collaboration entre la biologie et la psychanalyse »

Ce sont donc les limites de la connaissance rationnelle qui apparaissent comme autant de garanties de la liberté individuelle, comme si après une période où les sciences « exactes » apparaissaient inquiétante à cause de leurs certitudes (de 1975 à 1987 dans le corpus), on revenait à une conception plus limitée de leur rationalité. On constate alors que sur une même axiologie peuvent se greffer des valeurs ou des croyances qui évoluent dans le temps.

La présence de l'opposition « libre arbitre — domination » étant attestée dans le discours télévisuel à propos du cerveau, elle va maintenant autoriser l'analyse d'une séquence du documentaire «Les scientifiques répondent » qui, sans cela, serait restée assez difficile à interpréter. Voici tout d'abord la séquence dont seuls les plans les plus caractéristiques ont été conservés. Ils sont numérotés pour une meilleure compréhension des rapports texte/image.

Cette séquence se situe au milieu d'un documentaire d'une durée d'une heure, à la vingtsixième minute. Plusieurs expériences très techniques viennent d'être montrées et la dernière, portant sur l'influence de l'ionisation de l'air sur le comportement humain, prend pour « cobaye » une jeune fille dont un chercheur teste les réflexes auditifs.
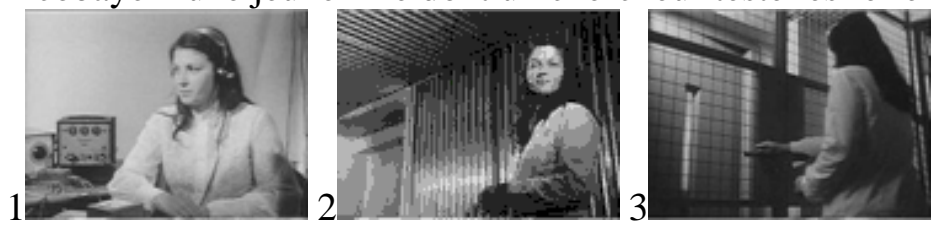

\footnotetext{
${ }^{23}$ Pour une analyse détaillée de l'histoire des relations de légitimation entre science et télévision, cf. Babou, Igor, « Science et télévision : la vulgarisation comme construction historique et sociale », Colloque «Émergences et continuité dans les recherches en information et en communication » organisé par la SFSIC, Paris, UNESCO, 11 janvier 2001 (publication à paraître)
} 
Chercheur off : «[...] il est maintenant prouvé que les irruption solaires jouent très nettement sur la vie. Et bien sûr, elles jouent très probablement [1] sur les comportements ».

[La jeune fille regarde la caméra puis part dans le couloir. Bruits de timbales d'orchestre.] [3] [La fille ouvre une grille d'ascenseur]
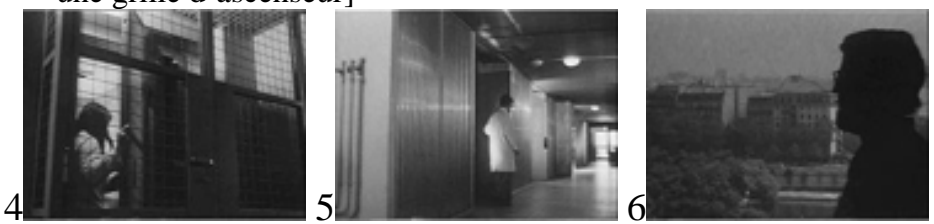

[4] [L'ascenseur descend] [5] [travelling avant lent dans le couloir. Son des timbales. Son de violons :

musique à tonalité lugubre] [6] [Lambert en contre-jour. Il ouvre une fenêtre]
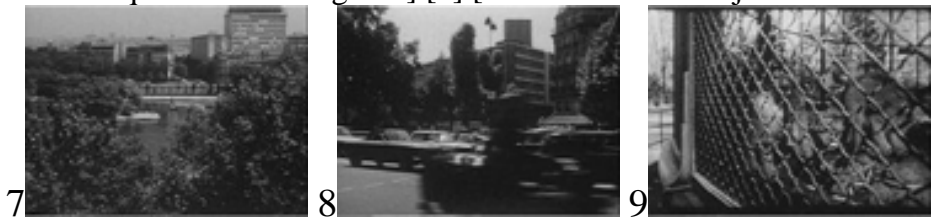

[7] [Son in de la rue] [8] [9] [Un zoo. Son in des singes dans leur cage]
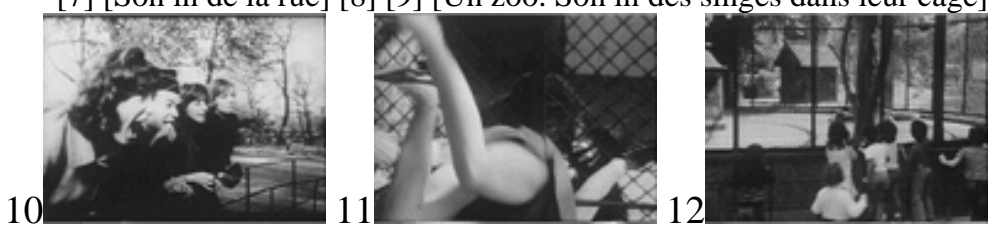

[10] [Cris du public du zoo. Une fille hurle. Des enfants lancent des objets aux singes (de la nourriture ou des pierres ?)] [11] [Cris des enfants]
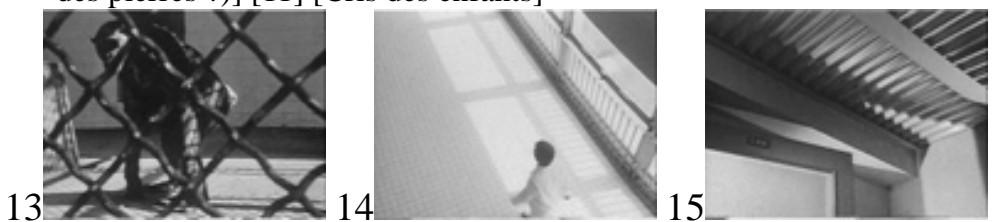

[13] [14] [Violons : musique à tonalité lugubre] [15] [Une porte s'ouvre. Lambert passe la porte accompagné du son d'un gong. Bruit d'un téléphone : quelqu'un compose un numéro]
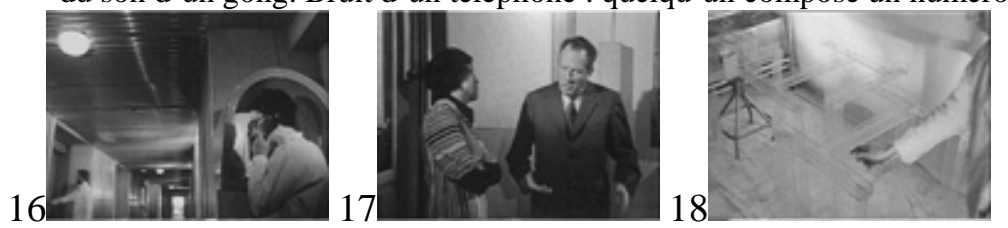

Son in de la fille au téléphone [16] : « Oui, heu, est-ce que je pourrais parler à Françoise s’il vous plaît ? Salut, c'est Josette à l'appareil. Oui, ça va, et tes révisions, ça avance ?... » [La conversation est couverte par une musique contemporaine orchestrale] [On entend les sons étouffés d'une interview : Journaliste : « Ils sont déterminés par quoi ? »Pr. Soulairac : «Et bien, les comportements instinctifs... »]

Son in de la fille au téléphone [16] : «Bon, ben alors, je te rappelle. Au revoir » [Elle raccroche] [17]

Son in de la journaliste : «Alors, que va-t-il se passer lorsque sur ces comportements fondamentaux va s'inscrire l'apprentissage?»

Son in Pr. Soulairac : «Vous posez un très gros problème parce que c'est ce qui revient à l'inné et ce qui revient à l'acquis. Alors d'une manière générale on a des techniques très simples pour étudier ce problème, à la fois chez l'adulte et à la fois chez le très jeune animal. Et la technique la plus classique est la technique du labyrinthe. Vous en avez un exemple [18] C'est un labyrinthe élevé dans lequel l'animal est placé et dans les branches duquel il doit retrouver son chemin [19]»

19

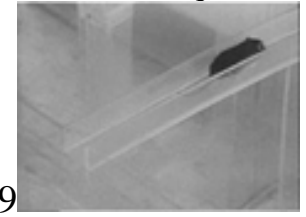

La séquence est étrange dans le contexte de ce documentaire. Elle intervient après une longue partie présentant des opérations techniques de la méthode scientifique. Or, elle ne se situe pas 
sur le même plan descriptif : ambiances sonores et musicales bizarres et même lugubres, images sombres, effets de contre-jour, nombreuses contre-plongées qui accentuent les déformations de l'architecture, superposition d'interviews off et de son in, hurlements des enfants, visages crispés et agressifs du public envers les singes, tout participe à une dramatisation qui inscrit une nette rupture dans le déroulement du documentaire. Ensuite, alors que la majorité des images du documentaire se situe à l'intérieur de l'université, la caméra fait une brusque incursion à l'extérieur, dans un zoo. Enfin, le montage alterne rapidement un nombre important de plans alors que le reste du reportage est plutôt constitué de longs plans séquences. On observe, en fait, une série d'opérations de métonymisation-métaphorisations dont on va montrer qu'elles ne peuvent s'expliquer que par condensation de l'axiologie « libre arbitre domination ». Par condensation, on reprend ici une terminologie que Metz $^{24}$ emprunte à la psychanalyse. Il s'agit à l'origine d'une contraction opérée au sein d'un « texte » attesté (rêve ou discours vigile), d'un «texte» long en un «texte» plus court. Metz prend l'exemple de la pratique du résumé pour montrer qu'une condensation est, avant tout, une réduction physique de la longueur d'un énoncé. Ceci, selon lui, ne constitue pas pour autant une négation de la portée symbolique de la condensation, mais en est au contraire la condition d'existence. Pour qu'une condensation se manifeste, dans un poème par exemple, il faut que « [...] différentes chaînes de pensée convergent en une sorte de court-circuit terminal, qui est le vers attesté, mais leur « combinaison » n'est pas explicitée, elle est, justement, condensée [...] : les chaînons intermédiaires ont disparu, le résultat est quelque peu illogique (bien que pleinement langagier) $\gg^{25}$

Dans la séquence du documentaire cité plus haut, on observe une série de métonymisationsmétaphorisations : les humains sont comme les rats, pris dans les couloirs d'une universitélabyrinthe. Les couloirs sont comme les parois élevées du labyrinthe. Le « comme»de l'équivalence métaphorique est obtenu par contiguïté métonymique au sein du montage. Cette métonymie opère tout d'abord au plan iconique : avec les nombreuses images de grilles (celle de l'ascenseur et celles du zoo, évoquant celles des cages des rats montrées tout au long du reportage), mais aussi avec les images des couloirs qui répondent aux parois vitrées du labyrinthe. Quant aux rainures des parois des couloirs de l'université, sur lesquelles la caméra s'attarde en d'inhabituelles contre plongées (en particulier le gros plan d'un plafond, en [15]), elles renforcent l'impression que les chercheurs sont enfermés derrière des grilles. C'est le cas aussi pour le plan [14] qui ressemble aux plans [18] et [19] : même plongée, même ambiance lumineuse (dominance du blanc) et même irruption d'un actant (un homme ou un rat) dont le mouvement contraste avec le fond. Ensuite, la métonymie opère au plan des actants : la jeune fille se déplace dans les couloirs comme le rat dans le labyrinthe. Cette séquence d'individus plus ou moins bien identifiés (la fille est-elle une « cobaye », une chercheuse, une étudiante ?) se déplaçant dans des couloirs sombres filmés en contre plongée est d'ailleurs récurrente dans le documentaire. Comme pour le rat, son comportement est observé par la caméra du réalisateur. Ce dernier se met ainsi dans la même position que les chercheurs par rapport aux rats. Qui observe qui ? Qui est sujet d'une expérimentation et qui en est l'objet ? Cette ambiguïté est renforcée par le passage à l'extérieur et les analogies comportementales entre les spectateurs (agités et bruyants) et les singes. L'alternance de plans du public et des singes, permet d'insister sur les grilles et sur l'ambiguïté de la situation topologique : qui est derrière une grille ? Le public ou les animaux ? Enfin, la métonymie opère également lorsqu'elle fait se succéder les plans les plus étranges du documentaire (les couloirs et le zoo) et les plans du labyrinthe, créant ainsi une causalité par succession temporelle. Tout ceci fait de l'université un immense labyrinthe. Partant, c'est l'ensemble de la société qui est, sous l'emprise de la

\footnotetext{
${ }^{24}$ METZ, Christian, Le signifiant imaginaire, Paris, Christian Bourgeois, 1993

${ }^{25}$ Ibid., p. 272.
} 
science, métaphorisée comme un lieu d'observation (le plan d'un chercheur en contre jour regardant la ville de haut semble l'attester et l'on retrouve le même procédé dans

«Temps $X$ » où la ville, filmée de haut, est sous la coupe d'un scientifique présenté comme un peu fou voire dangereux). Cette métaphorisation condense en quelques images l'axiologie « libre arbitre - domination ». Sans ce principe explicatif, comment comprendre l'ensemble de la séquence ? Ou plutôt, comment justifier l'analyse que l'on propose ?

La méthode qui consiste à étudier des régularités axiologiques dans un corpus d'émissions, et à les vérifier à travers l'intertextualité de corpus hétérogènes, permet de mieux comprendre les fondements du discours télévisuel : on fait en effet apparaître à la fois sa spécificité et ce qui le relie, en profondeur, à des représentations sociales cristallisées par l'histoire.

\section{L'esprit et le corps}

«Corps-esprit », il s'agit là d'une axiologie fondamentale dans la philosophie cartésienne, avec de profondes ramification dans la pensée contemporaine. Lorsque le corps et l'esprit sont appréhendés comme des entités radicalement séparées ou lorsqu'on insiste au contraire sur leur interdépendance, voire sur l'impossibilité d'une telle séparation conceptuelle, on pose la question du support matériel de la conscience. Au plan des théories biologiques, l'axiologie « esprit - corps » accompagne l'opposition « vitalisme — matérialisme ». Il s'agit d' un ensemble axiologique très général. On relève cette axiologie, de manière explicite, dans 15 émissions du corpus ${ }^{26}$.

Il y a une grande diversité dans les modes d'appropriation de cette axiologie par le discours télévisuel : elle peut être clairement verbalisée, ou apparaître à travers une série de métaphores tant verbales que visuelles. Voici tout d'abord quelques exemples qui explicitent verbalement la séparation de l'esprit et du corps. Ils sont extraits de la série «L'ordinateur cérébral » diffusée en 1987. Les deux premiers se situent en tout début d'émission, qui sont présentées par Pierre Desgraupes.

Pierre Desgraupes : «[...] il est vrai que le rôle de notre système nerveux est déterminant dans notre vie psychique. C'est même probablement le mystère le plus difficile à percer, dans notre condition humaine, que celui de cette frontière invisible qui sépare notre corps de notre esprit. Mais qu'il s'agisse du plus élémentaire de nos réflexes, ou de la plus sophistiquée de nos pensées, tous deux prennent leur origine dans la même activité corporelle, qui est un simple message qui court le long d'une cellule nerveuse [...] » Pierre Desgraupes : «Bien que rien ne nous permette matériellement de tracer une frontière entre l'esprit et le corps, on ne peut guère faire autrement que de regarder l'un et l'autre comme deux entités séparées. Dans cette série de films, nous avons concentré notre attention sur les aspects physiques de nos activités. Mais nous n'avons pas manqué non plus de souligner combien ce qu'il y a d'immatériel en nous, nos pensées, nos sensations, nos sentiments même, sont étroitement associés à l'activité de certaines de nos cellules. [...] ». Commentaire off : « [images de vieillards s'exerçant dans une salle de gymnastique] Conserver son corps en bonne forme physique prévient les effets les plus néfastes du vieillissement. Mais ne risque-t-on pas, à vouloir trop retarder les effets physiques de l'âge, de se retrouver avec un esprit plus détérioré que le corps ? [des vieillards jouent aux cartes]»

\footnotetext{
${ }^{26}$ Enquête sous un crâne (magazine avec plateau diffusé le 17.09.76 à 21h30 sur FR3) ; Portrait de l'univers : une révolution sous un crâne (documentaire diffusé le 15.10 .78 à $21 \mathrm{~h} 30$ sur Antenne 2); Les hémisphères ou les deux cerveaux (documentaire diffusé le 29.10.78 à 21h20 sur Antenne 2); La part des autres (documentaire diffusé le 15.10.1980 sur TF1); JT A2 18h30 Cerveau enfants (diffusé le 27.04.1982 à 18h36 sur Antenne 2); Le propre de l'homme : le cerveau (documentaire de la collection « Histoire de la vie » diffusé le 18.11.1982 à 22h50 sur TF1. Ce documentaire a été ensuite rediffusé trente fois sur TF1 entre 1988 et 1994) ; L'homme électronique (magazine de la collection « Dimension 3 » diffusé le 06.04.1987 à 22h36 sur FR3) ; Temps X : Professeur Delgado (diffusé le 02.05.1987 à $16 \mathrm{~h} 41$ sur TF1) ; L'ordinateur cérébral : les nerfs (diffusé le 01.06.1987 à 22h55 sur Antenne 2); L'ordinateur cérébral : l'intelligence (documentaire diffusé le 15.06.1987 à 22h17 sur Antenne 2) ; L'ordinateur cérébral : Vieillir (diffusé le 03.09.1987 à 23h55 sur Antenne 2) ; Un univers, l'homme (Collection « Génération 3 », séquence « Matière grise », diffusé le 18.01.94 à 9h53 sur France 3); Le fantôme de la Place Rouge (documentaire diffusé le 21.01.94 à 23h13 sur France 3) ; Corps et âme (magazine de la collection «Envoyé spécial » diffusé le 31.03.1994 à $20 \mathrm{~h} 57$ sur France 2) ; Nimbus : Jean-Didier Vincent (magazine diffusé le 18.11.94 à 23h25 sur France 3)
} 
Dans ce documentaire l'axiologie s'inscrit dans la narration : les effets du vieillissement sur le corps sont examinés en premier et ce n'est qu'ensuite que sont évoquées ses conséquences sur le cerveau. L'opposition est explicite dans le titre d'un documentaire diffusé en 1994 dans le cadre du magazine «Envoyé spécial » : intitulé « Corps et âme », il présente de nouvelles thérapies médicales visant à tenir compte de la psychologie des malades dans le traitement de leurs pathologies.

\section{La métaphore communicationnelle}

Dans ce premier groupe d'émissions, la communication est devenue l'une des disciplines légitimant le discours des journalistes. C'est parce que les patients vont se mettre à « communiquer» dans leur couple ou « communiquer» leur mal de vivre à un public lors d'un vaste psy show organisé dans un institut, qu'ils seront aptes à mieux gérer leur maladie. On constate aussi que pour caractériser le fonctionnement des nerfs, la référence aux théories de la communication organise un impressionnant cortège de métaphores avec des termes tels que «messages », « information », « code », « communication », « signaux », « langage », etc. De même, pour expliquer comment les cellules des nerfs « communiquent» entre elles et comment elles traitent un grand nombre de « messages », un documentaire présente les codes utilisés par les parieurs d'un champ de course anglais qui sont obligés de communiquer entre eux à distance par gestes. Et pour illustrer comment une molécule traverse l'espace qui sépare deux neurones, on montre un père de famille séparé de son fils par la largeur du champ de course. Le fils reçoit un appel sur le téléphone cellulaire de son père. Il traverse le champ de course, téléphone en main, et transmet ainsi l'appel. Un nombre important de métaphores utilisent ainsi une analogie entre les cellules nerveuses et les câblages téléphoniques ou électriques. Il apparaît clairement que la métaphore communicationnelle condense la distance conceptuelle entre les deux pôles de l'axiologie. Littéralement ainsi que visuellement, elle permet à la distance entre le hard (le cerveau comme entité biologique) et le soft (la pensée comme phénomène insaisissable), d'être franchie voire annulée. La télévision participe de l'incorporation sociale du champ de la communication : alors que les émissions antérieures à 1987 faisaient plutôt référence à des disciplines des sciences de la nature (en particulier la physiologie), on voit apparaître en 1987 des références aux techniques et aux théories de la communication. Il y a sans doute aussi une adéquation subtile entre ces théories et le rôle qu'elles jouent lorsqu'elles métaphorisent le fonctionnement cérébral : théories articulant le hard des machines à communiquer et le soft des messages de la culture. N'étaientelles pas bien adaptées pour condenser les deux pôles d'une axiologie qui s'appuie sur des dimensions comparables ?

\section{La métaphore mécaniste}

La métaphore qui condense la tension entre les deux pôles de l'axiologie n'est pas toujours celle de la communication. On relève en effet des métaphores mécanistes qui prennent en charge l'inscription de la conscience dans le registre du biologique ou du matériel. Le cerveau est alors qualifié de «machine », d' « horlogerie », etc. Dans un documentaire diffusé en 1978, un chercheur explique que les expériences de split-brain (une section du corps calleux qui réunit les deux hémisphères) pourraient doubler la capacité de traitement et de mémorisation du cerveau. Cette hypothèse, que le chercheur qualifie de «science fiction », est suivie par la séquence suivante :
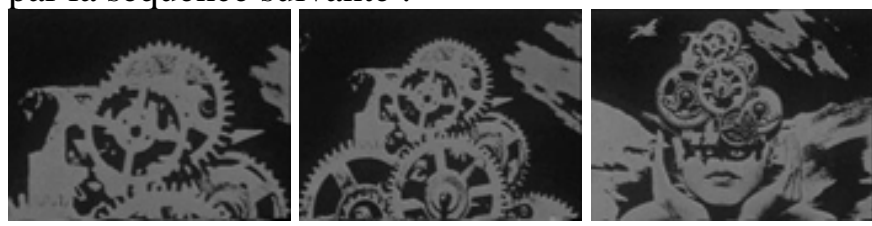
Commentaire off : «Quel rêve ! Penser deux fois plus, sentir deux fois plus, apprendre deux fois plus et, qui sait, produire deux fois plus. Pourtant, cerveau dédoublé ou pas, ce qu'on appelle la conscience reste la conscience. Alors, où se trouve-t-elle ? Est-il même pensable de la situer ? »

Le registre onirique de ces images permet, par juxtaposition d'un mécanisme d'horlogerie sur la partie supérieure d'un visage féminin, une condensation des deux pôles de l'axiologie. Verbalement, l'utilisation d'une terminologie productiviste sied à la conception contemporaine de la rationalité de la fin et des moyens. Mais, là aussi, le pôle soft de l'axiologie est repérable dans le refus explicite de localiser la conscience. On observe le même type de métaphore mécaniste lors d'une interview du professeur Baulieu, diffusée en 1994. Dans ce reportage, Baulieu est interrogé sur les relations entre les émotions et la chimie du cerveau. La métaphore mécaniste est alors filée aussi bien au plan de l'image qu'au plan syntagmatique. L'interview du scientifique est en effet précédée puis entrecoupée de plans montrant des pièces mécaniques :
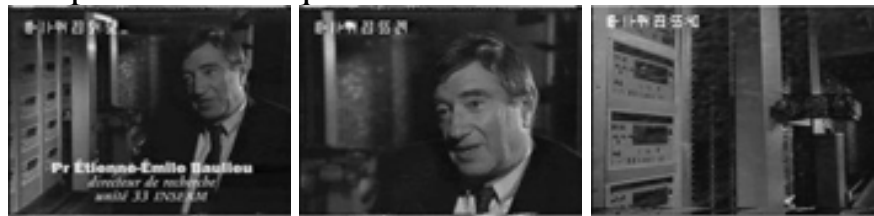

Pr Baulieu : « Les hormones comme les œstrogènes que les femmes connaissent bien, se transmettent aussi bien chez les humains que chez les poissons et... donc, dans beaucoup de formes de vie [...] » Commentaire off : « Nous partageons tous les mêmes mécanismes biologiques. [...]»

Dans ce cas, la métaphore mécaniste coexiste avec la métaphore communicationnelle. On voit en arrière plan de l'interview un matériel professionnel typique des régies de diffusion des télévisions : le bras mécanique d'un robot qui enchaîne automatiquement des cassettes vidéo lors de programmations gérées par ordinateur. Dans les plans précédant cette interview, la métaphore mécaniste est encore plus marquée dans la mesure où interviennent une série de gros plans sur les rouages du robot. La métaphore communicationnelle devient plus explicite lorsqu'un chercheur est interrogé dans un centre de contrôle audiovisuel autoroutier : environné d'une multitude d'écrans braqués sur une autoroute, il parle... du cerveau. Cette métaphore mécanico-communicationnelle n'est pas isolée dans le corpus. On la retrouve à l'identique dans un dessin animé où de petits personnages dirigent le corps depuis un centre de contrôle. Évoquant la technique autant que la communication, chargés dans le même temps de représenter la perception visuelle (écrans et caméras), les instruments audiovisuels fournissent à la télévision une métaphore de choix. Précisons toutefois que les métaphores exclusivement mécanistes sont les plus fréquentes lorsqu'il s'agit de condenser l'opposition « esprit - corps ». Les animaux-machines de Descartes ont, semble-t-il, fonctionné comme un modèle fécond. On notera du reste que dans les dictionnaires ou les textes épistémologiques, l'axiologie « esprit — corps » est régulièrement accompagnée de sa reformulation « raison - émotion », en particulier lorsqu'il s'agit de l'informatique ou de l'intelligence artificielle.

\section{Conclusion}

En fin de compte, c'est une partie de la culture et des idéologies de ces vingt dernières années que l'on a traversé en étudiant de simples métaphores. On a pu observer que le discours télévisuel à propos de science ne se contente pas de représenter des connaissances, mais qu'il s'inscrit dans le système axiologique des représentations de la rationalité qui nous a été légué par l'histoire. Ceci montre bien, au-delà de la médiatisation des débats sur telle ou telle découverte, que la structure d'une pensée de la rationalité agit en profondeur et organise certaines formes de la vulgarisation. Dans le même temps, la télévision ne constitue pas un reflet des représentations sociales puisqu'elle opère une sélection active inscrite dans une historicité : certaines valeurs associées à la rationalité évoluent au rythme de la légitimité sociale de la science, de l'émergence de certaines théories scientifiques, et d'un processus 
d'autoréférence dans lequel la télévision s'engage aujourd'hui en représentant abondamment ses techniques, ses matériels et les valeurs de la communication.

Les formes du discours télévisuel à propos de science ne semblent pas pouvoir être expliquées seulement à partir des intentions, des actions ou du «génie » de tel ou tel réalisateur ou animateur. Sans occulter l'importance du rôle des acteurs de la télévision, force est de constater que leurs productions s'inscrivent dans des logiques sociales et culturelles qui les dépassent, car elles correspondent à une circulation et à une structuration plus globale des idées. On peut ensuite penser que les caractéristiques des discours télévisuels conditionnent leur efficacité auprès du public. Même si l'on peut douter que la seule justification de la vulgarisation télévisuelle soit de «transmettre » des savoirs, il n'en reste pas moins important de comprendre les langages qu'elle met en œuvre. Enfin, le fonctionnement de la science s'inscrit aujourd'hui dans le débat public. Mais si les médias participent activement à la circulation sociale des connaissances, des opinions et des métaphores, on a bien vu que la question de la rationalité scientifique débordait largement du cadre des laboratoires : le système des axiologies de la rationalité constitue une matrice culturelle. C'est de ces constats que l'action sociale ou politique en matière de mise en culture des sciences doit se saisir si elle ne veut pas courir le risque de stigmatiser inutilement les médias, ou au contraire de faire une vaine apologie de leur rôle de médiateur culturel. C'est cette complexité des relations entre sciences, médias et société qu'il convient de saisir si l'on veut vraiment mettre en ouvre un «partage du savoir ». 